\title{
ON THE COMPLETENESS \\ OF A METHOD OF POTENTIALS \\ IN ELASTODYNAMICS
}

\author{
BY \\ RONALD Y. S. PAK (Department of Civil, Environmental and Architectural Engineering, \\ University of Colorado, Boulder, Colorado 80309-0428) \\ AND \\ MORTEZA ESKANDARI-GHADI (Civil Engineering Department, University of Science and \\ Technology of Mazandaran, Iran)
}

\section{A tribute to Eli Sternberg}

\begin{abstract}
In this paper, the theoretical foundation of a compact scalar potential method in three-dimensional classical elastodynamics is substantiated. Beginning with a derivation of two basic lemmas on the decomposition and integration of wave solutions and vector fields which are apt to be of interest to general mechanics and analysis, the treatment proceeds to a proof of the completeness of the proposed representation as well as its extension to non-zero body forces.
\end{abstract}

1. Introduction. In the classical three-dimensional theory of elasticity, the method of potentials has played a major role in the solution of many complex initial-boundary value problems (e.g., Love [1]; Fung [2]; Pao and Mow [3]; Achenbach [4]; Miklowitz [5]). Some of the most noteworthy potential functions include the Papkovich-Neuber solution and Somigliana-Galerkin vector for elastostatics, and Lamé's representation and the Kovalevshi-Iacovache-Somigliana solution for elastodynamics (see Truesdell [6]; Gurtin [7]). For many mechanics problems with $x_{3}$-convex domains, the more compact nonaxisymmetric augmented Love and Burgatti solutions for Navier's equations of equilibrium (e.g., see Tran-Cong [8]) and the scalar wave function approach in Pao and Mow [3] and Pak [9] for the equations of motion have been of comparable, if not even greater, practical interest. On the theoretical side, a fundamental question for all potential representations is their completeness (Sternberg [10]; Stippes [11]). In the case

\footnotetext{
Received May 24, 2007.

2000 Mathematics Subject Classification. Primary 74B05, 35Q72; Secondary 35L05.

Key words and phrases. Elasticity, completeness, potentials, mechanics, wave equation, vector calculus, elastodynamics, Helmholtz, solenoidal field, Laplacian.

E-mail address: pak@colorado.edu

E-mail address: ghadi@ustmb.ac.ir
} 
of the Papkovich-Neuber solution and Galerkin vector for elastostatics, the issue has been critically explored and established as in Mindlin [12], Sternberg and Gurtin [13] and Gurtin [14] for elastostatic problems (see also Freiberger [15]; Millar [16]; Tran-Cong [17]). On the more compact non-axisymmetric augmented Love and Burgatti solutions, their generality has also been validated as in Youngdahl [18] and Tran-Cong [8]. In classical elastodynamics, however, past attention has been focused mostly on Lamé and Kovalevshi-Iacovache-Somigliana solutions (see Sternberg [10]; Sternberg and Eubanks [19]; Sternberg and Gurtin [12]), leaving the completeness issue theoretically unsettled for some very potent wave functions.

To substantiate firmly the theoretical foundation for one such method of potentials for elastodynamics, a generalization to include body forces and the issue of completeness of the displacement potential representation employed in Pak [9] is addressed in this paper. With the aid of two basic lemmas on the decomposition and integration of wave solutions and vector fields whose relevance may go beyond this exploration, it will be shown that the proposed representation is indeed general for the solution of Navier's displacement equations of motion in suitably convex three-dimensional domains.

2. Notation and definitions. Consider a three-dimensional body $B$ to be regular in the sense of Kellogg [20], and let $\left(x_{1}, x_{2}, x_{3}\right)$ be the Cartesian coordinates for a material point $\mathbf{x} \in B$.

Notation. A vector field $\mathbf{u}(\mathbf{x}, t)$ is considered to be of class $C^{M, N}$ on $B \times\left(0, t_{0}\right)$ if it is continuous on $B \times\left(0, t_{0}\right)$ and its spatial derivatives up to the $M^{\text {th }}$ order and its time derivatives up to the $N^{t h}$ order are Hölder continuous on $B$ at any time $t \in\left(0, t_{0}\right)$.

Differential operators.

- $\nabla^{2}=\frac{\partial^{2}}{\partial x_{1}^{2}}+\frac{\partial^{2}}{\partial x_{2}^{2}}+\frac{\partial^{2}}{\partial x_{3}^{2}}$;

- $\square=\nabla^{2}-\frac{1}{c^{2}} \frac{\partial^{2}}{\partial t^{2}}$;

- $\square_{3}=\frac{\partial^{2}}{\partial x_{3}^{2}}-\frac{1}{c^{2}} \frac{\partial^{2}}{\partial t^{2}}$;

- $\nabla_{12}^{2}=\frac{\partial^{2}}{\partial x_{1}^{2}}+\frac{\partial^{2}}{\partial x_{2}^{2}}$.

Definition 2.1. The domain $B$ is called $x_{3}$-convex if every line segment parallel to the $x_{3}$-axis lies entirely in $B$ whenever its end points belong to $B$.

Definition 2.2. The region $S\left(x_{3}\right)=\left\{\left(x_{1}, x_{2}\right):\left(x_{1}, x_{2}, x_{3}\right) \in B\right\}$ is referred to as the cross section of $B$ at $x_{3}$.

Definition 2.3. If a vector field $\mathbf{u}(\mathbf{x}, t)$ of class $C^{2,2}$ on $B \times\left(0, t_{0}\right)$ satisfies the Navier equation of motion with a body-force density $\mathbf{b}(\mathbf{x}, t)$

$$
(\lambda+2 \mu) \nabla(\nabla \mathbf{u})-\mu \nabla \times(\nabla \times \mathbf{u})+\mathbf{b}=\rho \ddot{\mathbf{u}}
$$

or

$$
c_{1}^{2} \nabla(\nabla \mathbf{u})-c_{2}^{2} \nabla \times(\nabla \times \mathbf{u})+\frac{\mathbf{b}}{\rho}=\ddot{\mathbf{u}}
$$

where

$$
c_{1}^{2}=\frac{\lambda+2 \mu}{\rho}, \quad c_{2}^{2}=\frac{\mu}{\rho}
$$


with $\rho$ being the density, $\lambda$ and $\mu$ the Lamé constants of the homogeneous, isotropic, linearly elastic solid occupying $\mathrm{B}$, then $\mathbf{u}(\mathbf{x}, t)$ is called an elastic motion corresponding to $\mathbf{b}$.

\section{Mathematical preliminaries.}

Lemma 3.1. Existence of Solution of a Constrained 3D Wave Equation.

Suppose a function $f\left(x_{1}, x_{2}, x_{3}, t\right)$ is of class $C^{M, N}$ on $B \times\left(0, t_{0}\right), M, N \geq 2$ and $\square f=0$, there exists a scalar field $\Omega\left(x_{1}, x_{2}, x_{3}, t\right)$ of class $C^{M+2, N}$ on $B \times\left(0, t_{0}\right)$ such that $\square \Omega=0$ on $B \times\left(0, t_{0}\right)$ and $\nabla_{12}^{2} \Omega=f$ on any cross section $S\left(x_{3}\right)$ of a $x_{3}$-convex domain $B$.

Proof. With the condition of

$$
\nabla_{12}^{2} \Omega=\left(\frac{\partial^{2}}{\partial x_{1}^{2}}+\frac{\partial^{2}}{\partial x_{2}^{2}}\right) \Omega=f\left(x_{1}, x_{2}, x_{3}, t\right),
$$

the stipulation that

$$
\square \Omega=\frac{\partial^{2} \Omega}{\partial x_{1}^{2}}+\frac{\partial^{2} \Omega}{\partial x_{2}^{2}}+\frac{\partial^{2} \Omega}{\partial x_{3}^{2}}-\frac{1}{c^{2}} \frac{\partial^{2} \Omega}{\partial t^{2}}=0
$$

can be translated to the requirement of

$$
\square_{3} \Omega=-f .
$$

For a given $f\left(x_{1}, x_{2}, x_{3}, t\right)$ and $\left(x_{1}, x_{2}\right)$ in the domain, a particular solution $\Omega^{\prime}\left(x_{1}, x_{2}, x_{3}, t\right)$ for the foregoing inhomogeneous one-dimensional wave equation in $x_{3}$ and $t$ can be obtained by D'Alembert's double integration method (e.g., Weinberger [21]) so that

$$
\square_{3} \Omega^{\prime}=-f
$$

Likewise, because $\square f=0$,

$$
\square\left(\square_{3} \Omega^{\prime}\right)=\square_{3}\left(\square \Omega^{\prime}\right)=-\square f=0,
$$

i.e., $\square \Omega^{\prime}$ is a homogeneous solution of a one-dimensional wave equation in $x_{3}$ and $t$, and can be cast as

$$
\square \Omega^{\prime}=\nabla_{12}^{2} \Omega^{\prime}+\square_{3} \Omega^{\prime}=H\left(x_{1}, x_{2}, x_{3}-c t\right)+G\left(x_{1}, x_{2}, x_{3}+c t\right)
$$

where $H$ and $G$ are arbitrary functions or series of functions. Denoting

$$
g\left(x_{1}, x_{2}, x_{3}, t\right)=H\left(x_{1}, x_{2}, x_{3}-c t\right)+G\left(x_{1}, x_{2}, x_{3}+c t\right),
$$

it is evident from (3.3) and (3.6) that

$$
\nabla_{12}^{2} \Omega^{\prime}=g+f
$$

with

$$
\square_{3} g=0 .
$$

With the definition of $\Omega\left(x_{1}, x_{2}, x_{3}, t\right)=\Omega^{\prime}\left(x_{1}, x_{2}, x_{3}, t\right)+\Omega^{\prime \prime}\left(x_{1}, x_{2}, x_{3}, t\right)$ and the condition of (3.2), $\Omega^{\prime \prime}$ must satisfy

$$
\square \Omega^{\prime \prime}=-\square \Omega^{\prime}=-g\left(x_{1}, x_{2}, x_{3}, t\right) .
$$


To be consistent with (3.1) and (3.6), one must also demand that

$$
\nabla_{12}^{2} \Omega^{\prime \prime}=-g .
$$

On account of the characteristics of $g$ in (3.9), a particular solution to (3.11) can be given in the form of the logarithmic potential (Kellogg [20])

$$
\Omega^{\prime \prime}\left(x_{1}, x_{2}, x_{3}, t\right)=\frac{1}{2 \pi} \iint_{S} g\left(\xi_{1}, \xi_{2}, x_{3}, t\right) \log \left(\frac{1}{\sqrt{\left(x_{1}-\xi_{1}\right)^{2}+\left(x_{2}-\xi_{2}\right)^{2}}}\right) d \xi_{1} d \xi_{2}
$$

with the property that

$$
\square_{3} \Omega^{\prime \prime}=0 .
$$

By virtue of (3.6), (3.10), (3.8), (3.11), and (3.13), the existence of a scalar field $\Omega$ that satisfies

$$
\nabla_{12}^{2} \Omega=\nabla_{12}^{2}\left(\Omega^{\prime}+\Omega^{\prime \prime}\right)=f
$$

and

$$
\square \Omega=\square\left(\Omega^{\prime}+\Omega^{\prime \prime}\right)=0
$$

should thus be clear, completing the proof.

Lemma 3.2. Reduction of Solenoidal Vector Fields.

Suppose a vector field $\mathbf{u}$ of class $C^{N, M}, M, N \geq 2$ is generated as the curl of an arbitrary vector function $\boldsymbol{\Psi}$ of class $C^{N+1, M}$ on $B \times\left(0, t_{0}\right)$ with components $\left(\Psi_{1}, \Psi_{2}, \Psi_{3}\right)$ through

$$
\mathbf{u}\left(x_{1}, x_{2}, x_{3}, t\right)=\nabla \times \mathbf{\Psi}
$$

in a simply-connected region. Then there exist two scalar fields $\chi$ of class $C^{N+1, M}$ and $\eta$ of class $C^{N+2, M}$ such that $\mathbf{u}$ can be produced as

$$
\mathbf{u}\left(x_{1}, x_{2}, x_{3}, t\right)=\nabla \times \hat{\boldsymbol{\Psi}}
$$

where

$$
\hat{\mathbf{\Psi}}=\left(\chi \mathbf{e}_{3}\right)+\nabla \times\left(\eta \mathbf{e}_{3}\right) .
$$

Furthermore, if $\square \Psi=0$, then the two scalar fields $\chi$ and $\eta$ will also satisfy the wave equations

$$
\square \chi\left(x_{1}, x_{2}, x_{3}, t\right)=0, \quad \square \eta\left(x_{1}, x_{2}, x_{3}, t\right)=0 .
$$

Proof. Equating the components of (3.14) and (3.15), one may seek to write

$$
\Psi_{1}=\frac{\partial \eta}{\partial x_{2}}, \Psi_{2}=-\frac{\partial \eta}{\partial x_{1}}, \Psi_{3}=\chi
$$

for a given $\boldsymbol{\Psi}$. To ensure that $\Psi_{1}$ and $\Psi_{2}$ can be integrated for $\eta$, the necessary and sufficient condition of

$$
-\frac{\partial \Psi_{2}}{\partial x_{2}}=\frac{\partial \Psi_{1}}{\partial x_{1}}
$$

must be observed (see, e.g., Apostol [22]). For an arbitrary vector field $\mathbf{\Psi}$, such a requirement will generally not be satisfied. The incompatibility can, however, be removed in an equivalent representation by noting that the gradient of a sufficiently smooth scalar 
field $\alpha$ can always be added to $\Psi$ without affecting (3.14) by virtue of the identity $\nabla \times \nabla \alpha=0$. Accordingly, one may take

$$
\hat{\mathbf{\Psi}}=\mathbf{\Psi}+\nabla \alpha
$$

and define

$$
\hat{\Psi}_{1}=\Psi_{1}+\frac{\partial \alpha}{\partial x_{1}}=\frac{\partial \eta}{\partial x_{2}}, \hat{\Psi}_{2}=\Psi_{2}+\frac{\partial \alpha}{\partial x_{2}}=-\frac{\partial \eta}{\partial x_{1}}, \hat{\Psi}_{3}=\Psi_{3}+\frac{\partial \alpha}{\partial x_{3}}=\chi
$$

For the determination of $\eta$ in terms of $\hat{\boldsymbol{\Psi}}$, the compatibility condition of

$$
\frac{\partial^{2} \eta}{\partial x_{1} \partial x_{2}}=\frac{\partial^{2} \eta}{\partial x_{2} \partial x_{1}}
$$

translates to

$$
-\frac{\partial}{\partial x_{2}}\left(\Psi_{2}+\frac{\partial \alpha}{\partial x_{2}}\right)=\frac{\partial}{\partial x_{1}}\left(\Psi_{1}+\frac{\partial \alpha}{\partial x_{1}}\right)
$$

or

$$
\nabla_{12}^{2} \alpha=-\left(\frac{\partial \Psi_{1}}{\partial x_{1}}+\frac{\partial \Psi_{2}}{\partial x_{2}}\right)
$$

The existence of a scalar field $\alpha$ that satisfies (3.23) is assured by the theory of logarithmic potentials. This, in turn, confirms that $\eta$ and $\chi$ in (3.21) can be determined for a given $\Psi$.

For a vector potential $\boldsymbol{\Psi}$ that satisfies $\square \boldsymbol{\Psi}=0$, the field generated by the curl of $\hat{\boldsymbol{\Psi}}$ can be ensured to be identical to the one by $\boldsymbol{\Psi}$ with

$$
\square \hat{\mathbf{\Psi}}\left(x_{1}, x_{2}, x_{3}, t\right)=\mathbf{0}
$$

if

$$
\square(\nabla \alpha)=\nabla(\square \alpha)=\mathbf{0} .
$$

By virtue of $\square \Psi=0$ and the smoothness hypotheses, it should be evident that the inhomogeneous term of (3.23) satisfies the wave equation. Sufficient as a solution to (3.25), the existence of a scalar field $\alpha$ which satisfies $\square \alpha=0$ and (3.23) is thus assured by Lemma 3.1 which, in turn, affirms the existence of $\eta$ that satisfies (3.22). Noting that $\square \hat{\Psi}_{1}=\square \hat{\Psi}_{2}=0$, it also follows that $\frac{\partial}{\partial x_{1}} \square \eta=\frac{\partial}{\partial x_{2}} \square \eta=0$, i.e., $\square \eta$ is at least independent of $x_{1}$ and $x_{2}$. Allowing $\square \eta=k\left(x_{3}, t\right)$ in general, it is easy to see as in the case of (3.4) that one can add to $\eta$ a complementary function $\eta^{c}\left(x_{3}, t\right)$ such that $\square \eta^{c}=-k$ and $\frac{\partial}{\partial x_{1}} \eta^{c}=\frac{\partial}{\partial x_{2}} \eta^{c}=0$, thereby assuring the existence of a scalar field $\eta$ which satisfies $\square \eta=0$ and the first two relations in (3.21). With the explicit relation between $\chi$ and $\Psi_{3}$ in the last of (3.21) and $\square \boldsymbol{\Psi}=0$, it is evident that $\square \chi=0$, which completes the proof.

With the aid of the foregoing two lemmas, a complete theoretical foundation for the displacement potential representation in Pak [9] can be established as shown in the next section. 


\section{A method of displacement potentials in classical elastodynamics.}

THEOREM 4.1 (A general solution for classical elastodynamics with zero body forces). Let

$$
\mathbf{u}\left(x_{1}, x_{2}, x_{3}, t\right)=\nabla \phi+\nabla \times\left(\chi \mathbf{e}_{3}\right)+\nabla \times \nabla \times\left(\eta \mathbf{e}_{3}\right)
$$

where $\phi, \chi$ are of class $C^{3,2}$ and $\eta$ of class $C^{4,2}$ on $B \times\left(0, t_{0}\right)$ that satisfy

$$
\square_{1} \phi\left(x_{1}, x_{2}, x_{3}, t\right)=0, \square_{2} \chi\left(x_{1}, x_{2}, x_{3}, t\right)=0, \square_{2} \eta\left(x_{1}, x_{2}, x_{3}, t\right)=0,
$$

where

$$
\square_{\alpha}=\nabla^{2}-\frac{1}{c_{\alpha}^{2}} \frac{\partial^{2}}{\partial t^{2}}
$$

Then (4.1) is an elastic motion for $\mathbf{b}=\mathbf{0}$.

Proof. By direct substitution of (4.1) into (2.1) with $\mathbf{b}=\mathbf{0}$ and the stipulations in (4.2) (see also Pak [9]).

Theorem 4.2 (Completeness of the elastodynamic solution (4.1) for zero body forces). Let $\mathbf{u}$ be an elastic motion of class $C^{2,2}$ on $B \times\left(0, t_{0}\right)$ corresponding to zero body forces in a simply-connected, $x_{3}$-convex domain, and suppose that $\mathbf{u}(\cdot, \tau)$ and $\dot{\mathbf{u}}(\cdot, \tau)$ are continuous on $\bar{B}$ at some $\tau \in\left(0, t_{0}\right)$. Then there exist scalar fields $\phi, \chi$ of class $C^{3,2}$ and $\eta$ of class $C^{4,2}$ on $B \times\left(0, t_{0}\right)$ such that (4.1) and (4.2) hold.

Proof. Note that Lamé's solution in the form of a Helmholtz decomposition of

$$
\mathbf{u}\left(x_{1}, x_{2}, x_{3}, t\right)=\nabla \Phi+\nabla \times \mathbf{\Psi}
$$

is complete (e.g., Gurtin [7]) for elastodynamic states with zero body forces, with the scalar and vector potentials $\Phi$ and $\boldsymbol{\Psi}$ satisfying the homogeneous wave equations

$$
\begin{aligned}
& \square_{1} \Phi\left(x_{1}, x_{2}, x_{3}, t\right)=0, \\
& \square_{2} \Psi\left(x_{1}, x_{2}, x_{3}, t\right)=\mathbf{0},
\end{aligned}
$$

with

$$
\nabla \cdot \Psi\left(x_{1}, x_{2}, x_{3}, t\right)=0 .
$$

To show the completeness of the representation (4.1), it thus suffices to show that for every pair of Lamé potentials $\Phi$ and $\boldsymbol{\Psi}$, one can find three scalar potentials $\phi, \chi$, and $\eta$ such that (4.1) generates the same solution. To produce the gradient component of $\mathbf{u}$ with the foregoing objective, it is natural to take

$$
\Phi=\phi
$$

so that $\square_{1} \phi\left(x_{1}, x_{2}, x_{3}, t\right)=0$. Coupling it with Lemma 3.2 , one is thus assured of the possibility of writing (4.4) as

$$
\mathbf{u}\left(x_{1}, x_{2}, x_{3}, t\right)=\nabla \phi+\nabla \times \hat{\mathbf{\Psi}}
$$

where

$$
\begin{gathered}
\hat{\mathbf{\Psi}}=\left(\chi \mathbf{e}_{3}\right)+\nabla \times\left(\eta \mathbf{e}_{3}\right) \\
\hat{\Psi}_{1}=\frac{\partial \eta}{\partial x_{2}}, \hat{\Psi}_{2}=-\frac{\partial \eta}{\partial x_{1}}, \hat{\Psi}_{3}=\chi
\end{gathered}
$$


with

$$
-\frac{\partial \hat{\Psi}_{2}}{\partial x_{2}}=\frac{\partial \hat{\Psi}_{1}}{\partial x_{1}}
$$

and the existence of $\eta$ and $\chi$ as a function of $\boldsymbol{\Psi}$ with $\square_{2} \chi=0$ and $\square_{2} \eta=0$ as a result.

The foregoing results can be readily generalized to encompass the case of a non-zero body-force field $\mathbf{b}$, as described in the following theorems.

THEOREM 4.3 (A general solution for classical elastodynamics with non-zero body forces). Let $\mathbf{u}$ be generated by (4.1) with $\phi, \chi$ of class $C^{3,2}$ and $\eta$ of class $C^{4,2}$ on $B \times\left(0, t_{0}\right)$ that satisfy

$$
\begin{aligned}
& \square_{1} \phi\left(x_{1}, x_{2}, x_{3}, t\right)=\frac{\phi^{b}}{\rho c_{1}^{2}}, \\
& \square_{2} \chi\left(x_{1}, x_{2}, x_{3}, t\right)=\frac{\chi^{b}}{\rho c_{2}^{2}}, \\
& \square_{2} \eta\left(x_{1}, x_{2}, x_{3}, t\right)=\frac{\eta^{b}}{\rho c_{2}^{2}},
\end{aligned}
$$

with a body-force field of

$$
\mathbf{b}\left(x_{1}, x_{2}, x_{3}, t\right)=-\nabla \phi^{b}-\nabla \times\left(\chi^{b} \mathbf{e}_{3}\right)-\nabla \times \nabla \times\left(\eta^{b} \mathbf{e}_{3}\right),
$$

where $\phi^{b}, \chi^{b}$ are of class $C^{2,2}$ and $\eta^{b}$ is of class $C^{3,2}$ on $B \times\left(0, t_{0}\right)$. Then $\mathbf{u}$ is an elastic motion corresponding to $\mathbf{b}$.

Proof. By direct substitution of (4.1) into (2.1) with (4.13) and (4.14).

Theorem 4.4 (Completeness of the elastodynamic solution (4.1) for non-zero body forces). Let $\mathbf{u}$ be an elastic motion of class $C^{2,2}$ on $B \times\left(0, t_{0}\right)$ corresponding to a bodyforce field $\mathbf{b}$ which admits the Helmholtz decomposition as

$$
\mathbf{b}\left(x_{1}, x_{2}, x_{3}, t\right)=\nabla \Phi^{b}+\nabla \times \Psi^{b}
$$

where $\Phi^{b}$ and $\boldsymbol{\Psi}^{b}$ are of class $C^{2,2}$ in a simply-connected, $x_{3}$-convex domain, and suppose that $\mathbf{u}(\cdot, \tau)$ and $\dot{\mathbf{u}}(\cdot, \tau)$ are continuous on $\bar{B}$ at some $\tau \in\left(0, t_{0}\right)$. Then there exist scalar fields $\phi, \chi$ of class $C^{3,2}$ and $\eta$ of class $C^{4,2}$ on $B \times\left(0, t_{0}\right)$ such that (4.1), (4.13), and (4.14) hold.

Proof. On the basis of the first part of Lemma 3.2, it should be evident that the bodyforce field $\mathbf{b}(\mathbf{x}, t)$ of the form (4.15) can be expressed alternatively as (4.14). By virtue of the completeness of Lamé's solution for the case of non-zero body forces (Gurtin [7]) with its scalar and vector potentials satisfying

$$
\begin{aligned}
& \square_{1} \Phi=\frac{\Phi^{b}}{\rho c_{1}^{2}}, \\
& \square_{2} \Psi=\frac{\Psi^{\mathbf{b}}}{\rho c_{2}^{2}},
\end{aligned}
$$

one may define and obtain $\phi, \chi$, and $\eta$ in terms of $\Phi$ and $\boldsymbol{\Psi}$ by means of (4.8) and (4.11). Owing to their direct relations to $\Phi$ and $\Psi_{3}, \phi$ and $\chi$ will satisfy the inhomogeneous wave 
equations (4.13a) and (4.13b), respectively. As

$$
\begin{gathered}
\square_{2}\left(\frac{\partial \eta}{\partial x_{1}}\right)=-\square_{2} \Psi_{2}=-\frac{\Psi_{2}^{b}}{\rho c_{2}^{2}}=\frac{1}{\rho c_{2}^{2}} \frac{\partial \eta^{b}}{\partial x_{1}}, \\
\square_{2}\left(\frac{\partial \eta}{\partial x_{2}}\right)=\square_{2} \Psi_{1}=-\frac{\Psi_{1}^{b}}{\rho c_{2}^{2}}=\frac{1}{\rho c_{2}^{2}} \frac{\partial \eta^{b}}{\partial x_{2}}
\end{gathered}
$$

it follows that

$$
\frac{\partial}{\partial x_{1}}\left(\square_{2} \eta-\frac{1}{\rho c_{2}^{2}} \eta^{b}\right)=\frac{\partial}{\partial x_{2}}\left(\square_{2} \eta-\frac{1}{\rho c_{2}^{2}} \eta^{b}\right)=0 .
$$

By means of a deduction similar to the last part of the proof of Lemma 3.2, one can thus take without loss of generality that there exists a scalar field $\eta$ such that $(4.13 \mathrm{c})$ and (4.11) hold.

5. Conclusion. In this paper, a proof of the completeness and generalization of a method of displacement potentials to include body forces in classical elastodynamics is presented. By virtue of two fundamental lemmas which should be of relevance to general mechanics analysis and theoretical formulations, the possibility of reducing the four scalar functions in the Helmholtz-Lamé vector decomposition explicitly to three is also illuminated. The versatility and convenience of the proposed displacement functions and related representations in conjunction with orthogonal curvilinear coordinates and integral transform methods has been well illustrated in a number of fundamental as well as advanced boundary value problems (e.g., see Pao and Mow [3], Miklowitz [5], Pak and Guzina [23]).

6. Acknowledgement. The partial support from the National Science Foundation through CMS-0201353 during this work is gratefully acknowledged.

\section{REFERENCES}

[1] Love, A. E. H., A Treatise on the Mathematical Theory of Elasticity, 4th ed., Dover, New York, 1944. MR0010851 (6:79e)

[2] Fung, Y. C., Foundations of Solid Mechanics, Prentice-Hall, Englewood Cliffs, New Jersey, 1965.

[3] Pao, Y. H. and Mow, C. C., Diffraction of Elastic Waves and Dynamic Stress Concentrations, Crane Russak, New York, 1971.

[4] Achenbach, J. D., Wave Propagation in Elastic Solids, North-Holland, Amsterdam, 1973.

[5] Miklowitz, J., The Theory of Elastic Waves and Wave Guides, North-Holland, Amsterdam, 1978. MR515886 (80d:73001)

[6] Truesdell, C., Invariant and complete stress functions for general continua. Arch. Ration. Mech. Anal., 4 (1959), 1-29. MR0122083 (22:12810)

[7] Gurtin, M. E., The Linear Theory of Elasticity, Mechanics of Solids, Vol. II (ed. Truesdell), SpringerVerlag, Berlin, 1972, 1-295.

[8] Tran-Cong, T., On the completeness and uniqueness of Papkovich-Neuber and the nonaxisymmetric Boussinesq, Love and Burgatti solutions in general cylindrical coordinates. J. Elasticity, 36 (1995), 227-255. MR1318389 (96a:73020)

[9] Pak, R. Y. S., Asymmetric wave propagation in an elastic half-space by a method of potentials. J. Appl. Mech., ASME, 54(1) (1987), 121-126.

[10] Sternberg, E., On the integration of the equation of motion in the classical theory of elasticity. Arch. Ration. Mech. Anal., 6 (1960), 34-50. MR0119547 (22:10308)

[11] Stippes, M., Completeness of Papkovich potentials. Quart. Appl. Math., 26 (1969), 477-483. MR0239801 (39:1158) 
[12] Mindlin, R. D., Note on the Galerkin and Papkovich stress functions. Bull. Amer. Math. Soc., 42 (1963), 373-376.

[13] Sternberg, E. and Gurtin, M. E., On the completeness of certain stress functions in the linear theory of elasticity. Proc. Fourth U.S. Nat. Congr. Appl. Mech. (1962), pp. 793-797. MR0159454 $(28: 2671)$

[14] Gurtin, M. E., On Helmholtz's theorem and the completeness of the Papkovich-Neuber stress functions in infinite domains. Arch. Ration. Mech. Anal., 9 (1962), 225-233. MR0187467 (32:4917)

[15] Freiberger, W., On the solution of the equilibrium equations of elasticity in general curvilinear coordinates. Austral. J. Sci., A2 (1949), 483-492. MR0038821 (12:457d)

[16] Millar, R. F., On the completeness of Papkovich potentials. Quart. Appl. Math., 41 (1984), 385-393. MR724050 (85e:73009)

[17] Tran-Cong, T., On the completeness of the Papkovich-Neuber solution. Quart. Appl. Math., 47 (1989), 645-659. MR1031682 (91e:73022)

[18] Youngdahl, C. K., On the completeness of a set of stress functions appropriate to the solution of elasticity problems in cylindrical coordinates. Int. J. Engrg. Sc., 7 (1969), 61-70. MR0239803 (39:1160)

[19] Sternberg E. and Eubanks, R. A., On stress functions for elastokinetics and the integration of the repeated wave equation. Quart. Appl. Math., 15 (1957), 149-153. MR0091657 (19:1000d)

[20] Kellogg, O. D., Foundation of Potential Theory, Dover, New York, 1953.

[21] Weinberger, H. F., A First Course in Partial Differential Equations with Complex Variables and Transform Methods, Wiley, New York, 1965. MR0180739 (31:4969)

[22] Apostol, T. M., Mathematical Analysis, Addison-Wesley, Reading, Massachusetts, 1957. MR0087718 (19:398e)

[23] Pak, R. Y. S. and Guzina, B. B., Three-dimensional Green's functions for a multi-layered half-space by displacement potentials. J. Engrg. Mech. ASCE, 128(4) (2002), 449-461. 